\title{
EFICIENCIA PERSISTENTE Y TRANSITORIA EN LA PRODUCCIÓN DE CARNE DE CERDO EN EUROPA: UN ANÁLISIS DE FRONTERA ESTOCÁSTICA
}

\author{
Juan Cabas Monje ${ }^{a^{*}}$, Bouali Guesmi $^{\text {}^{*}}$, Amer Ait Sidhoum ${ }^{\mathrm{c}}$,José María Gil ${ }^{\mathrm{b}}$ \\ ${ }^{a}$ Facultad de Ciencias Empresariales, Departamento de Gestión Empresarial, Grupo de Investigación en \\ Agronegocios. Universidad del Bío-Bío, Región de Ñuble, Chile. jcabas@ubiobio.cl. ${ }^{b}$ Center for Agro- \\ Food Economics and Development (CREDA-UPC-IRTA), Castelldefels, España. bouali.guesmi@upc.edu; \\ chema.gil@upc.edu.'Technische Universität München, Department of Agricultural Production and \\ Resource Economics, Alte Akademie 14, 85354 Freising, Germany
}

\begin{abstract}
Resumen
La producción de carne de porcino tiene una gran importancia en las economías del sector agropecuario de Dinamarca, Francia, España y Polonia. Esta relevancia genera el interés por estudiar la eficiencia técnica de este sistema productivo en los cuatro países. El presente trabajo estima la eficiencia persistente, transitoria y total mediante un modelo de frontera estocástica del tipo cuatro componentes aplicado a muestras de productores porcinos de los cuatro países, para el periodo 2010-2015. Los resultados muestran que la eficiencia persistente es la más relevante para España, Francia y Dinamarca, con valores de 94\%, $89 \%$ y $87 \%$ respectivamente. La eficiencia transitoria es la componente más importante para Polonia alcanzando $93 \%$ de eficiencia. La eficiencia total muestra el valor máximo para Dinamarca con76\% y el menor para España con $72 \%$. Estos resultados entregan importante información para la toma de decisiones. Polonia debe implementar políticas que permitan mejorar la estructura de su sistema productivo, mientras que los otros países deben tratar de generar incentivos de corto plazo para mejorar su eficiencia transitoria.
\end{abstract}

Palabras clave: Eficiencia persistentey transitoria, frontera estocástica, producción porcina

\section{Introducción}

En la Unión Europea, la producción porcina es liderada por Alemania con un 22\%, seguida por España con el 20\%, a continuación, está Francia con un 9\% y luego Polonia en el cuarto lugar con un 8\%, a lo anterior, se suma Dinamarca con el 6\% de la producción europea de carne de cerdo para el año 2019 (Eurostat, 2020). Dada las similitudes y diferencias entre los sistemas productivos de carne de cerdo de estos países resulta relevante poner atención en su evolución para entender el desarrollo de la industria de la carne porcina en la región. Los cambios experimentados en los sistemas de producción, el rápido desarrollo de la industria de la carne porcina en estos cuatro países y su efecto en la economía local y nacional ponen en relevancia el estudio de sus dinámicas y de sus niveles de eficiencia productiva. Para estimar la eficiencia productiva se ha utilizado el modelo de frontera estocástica del tipo cuatro componentes (Colombi et al., 2014; , Kumbhakar et al., 2014; Tsiona y Kumbhakar, 2014), tomando en cuenta en forma simultánea las ineficiencias persistente (largo plazo), la ineficiencia transitoria (corto plazo), la heterogeneidad no observada y los shocks aleatorios. Esta aproximación incorpora el hecho que la ineficiencia puede arribar de diferentes fuentes y no todas son controladas por los productores y que es esencial controlar heterogeneidad de productores junto con distinguir entre eficiencia transitoria (gestionada individualmente) y la eficiencia persistente. En la práctica, según Lai y Kumbhakar (2018), esta separación entre eficiencia persistente y transitoria tiene mucha importancia debido a que ambas tiene diferentes implicancias respecto a las políticas a aplicar en el sector. El propósito de este trabajo es estimar las eficiencias total, persistente y transitoria en la producción porcina en cuatro países de Europa. Este artículo es uno de los primeros intentos con este propósito.

\section{Materiales y Métodos}

Para lograr el objetivo del estudio aplicamos el modelo propuesto por Badunenko y Kumbhakar (2016) y Filippini y Greene (2016). Para ello se plantea una forma funcional tipo Cobb-Douglas: $\ln \left(y_{i t}\right)=\beta_{0}+$ $\sum_{k=1}^{p} \beta_{k} \ln \left(x_{i t}\right)+\mu_{i}+v_{i t}-\eta_{i}-u_{i t}$ Donde, $\mathrm{i}=1,2, \ldots, \mathrm{n}$ representa $\mathrm{n}$ productores en la muestra $\mathrm{y} \mathrm{t}=$ $1,2, \ldots, \mathrm{T}$, los años $\mathrm{T}$. Y representa la producción (en euros), del productor i en el periodo t, X representa los factores productivos, y $\beta$ indica los parámetros desconocidos. La componente aleatoria del efecto del productor $\mu_{i}$ captura la heterogeneidad no observada, $v_{i t}$ es una variable aleatoria que representa el efecto de los shocks exógenos en la producción porcina. Las ineficiencias quedan representadas por la variable aleatoria no-negativa $u_{i t}$ para la ineficiencia transitoria, y por la variable aleatoria no negativa $\eta_{i}$ para la ineficiencia persistente.

Para el análisis empírico, se utilizan datos de producción porcina facilitados por FADN (Farm Accountancy Data Network) para el período 2010 al 2015. Estos datos corresponden a paneles no balaceados de productores de Dinamarca, España, Francia y Polonia donde al menos el 85\% del ingreso es originado de 
la producción porcina. La variable producción de cerdos esta medida como el ingreso total en euros del 2015. Se definen cinco factores productivos, el capital1, el capital2, el trabajo, alimento y otros insumos. El capital1 comprende edificios, equipamiento, maquinas, tractores, entre otros. El capital2 incluye el número de cerdos presentes en el sistema (promedio anual) que son convertidos en unidades de ganado expresados en euros. El trabajo se describe como el trabajo total expresado en AWU, el alimento medido en euros y otros insumos comprende otros costos específicos del ganado (veterinario, compra ocasional de animales, marketing más gastos generales en euros). Los valores están expresados en euros del año 2015. Finalmente, se incorpora la variable tiempo o tendencia para incorporar el efecto de la tecnología en la producción.

\section{Resultados}

En esta sección presentamos los principales resultados de la estimación de los modelos de frontera estocástica. Podemos observar en la tabla 1 que los signos de las elasticidades de producción son positivos y significativos estadísticamente al nivel del $1 \%$ o del $5 \%$ para los cuatro modelos, excepto el capital1 para España.

Tabla 1. Resultados Modelo de Cuatro Componentes

\begin{tabular}{|l|r|r|r|r|}
\hline Parámetros & \multicolumn{1}{|c|}{ Dinamarca } & \multicolumn{1}{c|}{ España } & \multicolumn{1}{c|}{ Francia } & \multicolumn{1}{c|}{ Polonia } \\
\hline Constante & $11.782 * * *(0.689)$ & $-29.029 * * *(10.252)$ & $12.665 * * *(5.584)$ & $-45.776 * * *(9.880)$ \\
\hline LN(capital1) & $0.036 * * *(0.001)$ & $0.018(0.014)$ & $0.019 * *(0.007)$ & $0.154 * * *(0.032)$ \\
\hline Ln(capital2) & $0.265 * * *(0.005)$ & $0.229 * * *(0.029)$ & $0.195 * * *(0.039)$ & $0.147 * * *(0.028)$ \\
\hline Ln(feed) & $0.516 * * *(0.001)$ & $0.537 * * *(0.023)$ & $0.538 * * *(0.035)$ & $0.448 * * *(0.031)$ \\
\hline Ln(labor) & $0.034 * * *(0.002)$ & $0.088 * * *(0.028)$ & $0.082 * * *(0.019)$ & $0.147 * * *(0.037)$ \\
\hline Ln(other) & $0.208 * * *(0.003)$ & $0.138 * * *(0.023)$ & $0.199 * * *(0.029)$ & $0.147 * * *(0.029)$ \\
\hline trend & $-0.006 * * *(0.0003)$ & $0.015 * * *(0.005)$ & $-0.006 * *(0.003)$ & $0.024 * * *(0.005)$ \\
\hline Log(lambda) & $7.228 * * *(0.004)$ & $0,234 *(0.124)$ & $0.557 * *(0.131)$ & $-2,609(0.239)$ \\
\hline Log(sigma) & $-2.239 * * *(0.174)$ & $-1.438 * * *(0.118)$ & $-2.242 * * *(0.179)$ & $-2.004 * * *(0.102)$ \\
\hline Random noise & $-2.738 * * *(0.006)$ & $-2.373 * * *(0.416)$ & $-2.901 * * *(0.281)$ & $-2.557 * * *(0.328)$ \\
\hline $\begin{array}{l}\text { Persistent } \\
\text { Inefficiency }\end{array}$ & $-2.738 * * *(0.006)$ & $-5.156 * * *(2.608)$ & $-2.973 * * *(0.247)$ & $-1.798 * * *(0.228)$ \\
\hline
\end{tabular}

Nota: Error estándar en paréntesis ()$, *(\mathrm{p}<0.1), * *(\mathrm{p}<0.05), * * *(\mathrm{p}<0.01)$

En general, los coeficientes estimados muestran para los cuatro países un comportamiento equivalente en cuanto a la significancia estadística y al orden de importancia de los factores productivos. El efecto más importante en todos los modelos corresponde al alimento con valores sobre 0.5 para España, Dinamarca y Francia. Le sigue en importancia el capital 2 con elasticidades de producción de 0.14 en Polonia, hasta 0.26 en Dinamarca. Por el contrario, el efecto de menor importancia en todos los modelos es el capital1. Los componentes de ruido aleatorio e ineficiencia persistente son estadísticamente significativos en todos los modelos. Las elasticidades de producción presentan valores levemente superiores a uno para España, Dinamarca y Francia, y bajo uno para Polonia. Esto indicaría que Polonia presenta rendimientos decrecientes de escala y los demás, rendimientos levemente crecientes de escala. En cuanto a la variable tiempo (proxy de tecnología), esta es significativa estadísticamente en las cuatro fronteras estimadas con signo positivo para España y Polonia y negativo para Dinamarca y Francia. Esto último indica que la producción media disminuyo en el período de estudio.

La Tabla 2 presenta los valores de las eficiencias persistentes, transitoria y total para los modelos estimados. La eficiencia media total estimada de todos los países está sobre 0.70, con el mayor valor para Dinamarca, alcanzando 0.756 y el menor para España con 0.718. Debemos recalcar que no podemos comparar directamente estos valores, ya que los modelos entregan estimaciones de eficiencia técnica relativas a las condiciones particulares de cada país. La eficiencia media total en Dinamarca ha disminuido de 0.775 hasta 0.771, situación parecida se encontró para Francia. Esto en parte puede explicar el signo negativo del coeficiente de la variable tendencia para ambos países. La eficiencia transitoria muestra valores dispares para los cuatro países, con el valor más alto de 0.927 para Polonia y el más bajo para España con 0.759. Esto significa que los productores porcinos polacos trabajan en el límite superior de la eficiencia de corto plazo, es decir son muy eficientes en términos relativos a sus pares.

Tabla 2. Eficiencias totales, transitorias y persistentes

\begin{tabular}{|l|l|l|l|l|}
\hline País & Media & SD & Mínimo & Máximo \\
\hline España & & & & \\
\hline
\end{tabular}




\begin{tabular}{|l|c|c|c|c|}
\hline Total & 0.718 & 0.032 & 0.446 & 0.826 \\
\hline Transitoria & 0.759 & 0.034 & 0.493 & 0.869 \\
\hline Persistente & 0.946 & 0.002 & 0.943 & 0.952 \\
\hline Francia & & & & 0.785 \\
\hline Total & 0.736 & 0.019 & 0.595 & 0.890 \\
\hline Transitoria & 0.829 & 0.019 & 0.689 & 0.908 \\
\hline Persistente & 0.888 & 0.009 & 0.860 & 0.816 \\
\hline Polonia & & & & 0.936 \\
\hline Total & 0.751 & 0.026 & 0.678 & 0.876 \\
\hline Transitoria & 0.927 & 0.003 & 0.912 & 0.835 \\
\hline Persistente & 0.810 & 0.027 & 0.739 & 0.934 \\
\hline Dinamarca & & & & 0.896 \\
\hline Total & 0.756 & 0.049 & 0.582 & 0.674 \\
\hline Transitoria & 0.872 & 0.053 & 0.852 & \\
\hline Persistente & 0.878 & 0.012 & & \\
\hline
\end{tabular}

Fuente: Elaboración propia

En cambio, en España a pesar que en general los productores son eficientes, todavía tienen un espacio de mejora en su gestión de año en año, de tal forma de remover ineficiencia de corto plazo. Francia y Dinamarca alcanzan valores medios de eficiencia transitoria de 0.829 y 0.872 , respectivamente. A pesar de los altos niveles de eficiencia, al igual que España, los productores de estos dos países todavía pueden mejorar su gestión y disminuir la ineficiencia de corto plazo. En el caso de la eficiencia persistente, estructural o de largo plazo, el mayor valor lo alcanza España con una eficiencia media de 0.946, en tanto que el menor valor corresponde a Polonia con 0.810. Esto indica que, en España, a pesar que los productores necesitan mejorar su gestión de año en año, la estructura general del sistema productivo porcino español permite un buen rendimiento y un nivel de eficiencia persistente alto, lo que también afecta positivamente al nivel de eficiencia técnica total. Se supone que en una estructura plenamente competitiva no debe existir ineficiencia persistente, de tal forma que esta cifra indica que el sistema productivo español es altamente competitivo. En cambio, Polonia necesita incorporar políticas adecuadas, junto con cambios en la gestión, para mejorar la estructura del sistema productivo porcino de tal forma de hacerlo más competitivo, lo que permitirá aumentar la eficiencia persistente. En Francia y Dinamarca la eficiencia persistente tiene una media de 0.887 , y 0.878 , respectivamente. En el caso francés este valor medio es aproximadamente 6 puntos porcentuales mayor que la eficiencia transitoria, lo que indicaría que la estructura del sector porcino francés está bien desarrollada y que los esfuerzos deben estar encaminados a mejorar la gestión individual de corto plazo del productor francés sin dejar de lado el desarrollo de políticas que permitan aumentar aún más la alta eficiencia persistente. En tanto que, para Dinamarca esta eficiencia es similar a la eficiencia transitoria, y su valor también indica una estructura del sector porcino adecuada para el desarrollo productivo, pero que todavía da espacio a mejoras en políticas que permitan aumentar la eficiencia persistente y por ende la eficiencia total de los productores porcinos de Dinamarca.

\section{Conclusiones}

En este trabajo investigamos sobre la eficiencia técnica en producción porcina para cuatro países de Europa. Desde las evidencias empíricas podemos extraer algunas consideraciones generales sobre política y gestión. Primero, España y Francia deben mejorar la eficiencia transitoria, sobretodo la focalización se debe realizar en aquellos productores que presentan niveles muy bajos de eficiencia transitoria. La eficiencia transitoria o de corto plazo es gestión individual de año en año, por lo que principalmente es responsabilidad de cada productor. Se sugiere diseñar incentivos de corto plazo para apoyar a los productores para mejorar la eficiencia temporal o transitoria. Segundo, Polonia debe enfocar sus esfuerzos en mejorar la estructura de su sistema productivo porcino, de tal manera de aumentar la eficiencia persistente. Se sugiere que la autoridad desarrolle políticas que disminuyan la fragmentación aumentando la asociatividad, que permitan mayor integración vertical aumentando la colaboración entre los diferentes niveles de la cadena de valor de la producción porcina. Tercero, a pesar de los altos valores de eficiencia transitoria y persistente que presenta Dinamarca, todavía queda una ventana para mejorar en ambas.

\section{Referencias}

Badunenko, O. y Kumbhakar, S. C. (2016). "When, where and how to estimate persistent and transient efficiency in stochastic frontier panel data models". European Journal of Operational Research, 255(1): 272-287.

Colombi, R., Kumbhakar, S. C., Martini, G. y Vittadini, G. (2014). "Closed-skew normality in stochastic frontiers with individual effects and long/short-run efficiency". Journal of Productivity Analysis, 42(2): 123-136. 
Eurostat. (2020). Agricultural production - livestock and meat - Statistics Explained. https://ec.europa.eu/eurostat/statisticsexplained/index.php?title=Agricultural_production_livestock _and_meat\&oldid=427096\#Livestock_population

Filippini, M. y Greene, W. (2016). "Persistent and transient productive inefficiency: a maximum simulated likelihood approach". Journal of Productivity Analysis, 45(2): 187-196.

Kumbhakar, S. C. y Lien, G. (2017). "Yardstick regulation of electricity distribution - Disentangling shortrun and long-run inefficiencies". Energy Journal, 38(5):17-37.

Kumbhakar, S. C., Lien, G. y Hardaker, J. B. (2014). "Technical efficiency in competing panel data models: A study of Norwegian grain farming". Journal of Productivity Analysis, 41(2):321-337.

Kumbhakar, S. C. y Parmeter, C.F. (2019). "Implementing Generalized Panel Data Stochastic Frontier Estimators". In Panel Data Econometrics.

Larue S., Abildtrup J. y Schmitt B. (2008). "Modelling the Spatial Structure of Pig Production in Denmark". $12^{\text {th }}$ Congress of the European Association of Agricultural Economists - EAAE 2008.

Roguet C., Gaigné C., Cariou S., Carlier M., Chatellier V., Chenut R., Daniel K. y Perrot C. (2015). Spécialisation territoriale et concentration des productions animales européennes: état des lieux et facteurs explicatifs. Productions Animales, INRA Éditions, 28 (1):5-22 